\title{
Controlled Synthesis of Ordered Mesoporous Carbon-Cobalt Oxide Nanocomposites with Large Mesopores and Graphitic Walls
}

Zhengren Wang ${ }^{\dagger \neq}$, Yongheng Zhu ${ }^{\dagger \S_{\ddagger}}$, Wei Luo ${ }^{\ddagger}$, Yuan Ren ${ }^{\dagger}$, Xiaowei Cheng ${ }^{\dagger}$, Pengcheng Xü, Xinxin $\mathrm{Li}^{\#}$, Yonghui Deng ${ }^{*}{ }^{* \#}$, Dongyuan Zhao ${ }^{\dagger}$

$\dagger$ Department of Chemistry, State Key Laboratory of Molecular Engineering of Polymers, Shanghai Key Laboratory of Molecular Catalysis and Innovative Materials, and iChEM, Fudan, Shanghai 200433, China

${ }^{\S}$ College of Food Science and Technology, Shanghai Ocean University, Shanghai 201306, China

${ }^{\ddagger}$ College of Materials Science and Engineering, Donghua University, Shanghai

201620, China

\# State Key Lab of Transducer Technology, Shanghai Institute of Microsystem and

Information Technology, Chinese Academy of Sciences, Shanghai 200050, China.

${ }^{\ddagger}$ These authors contribute equally to this study 

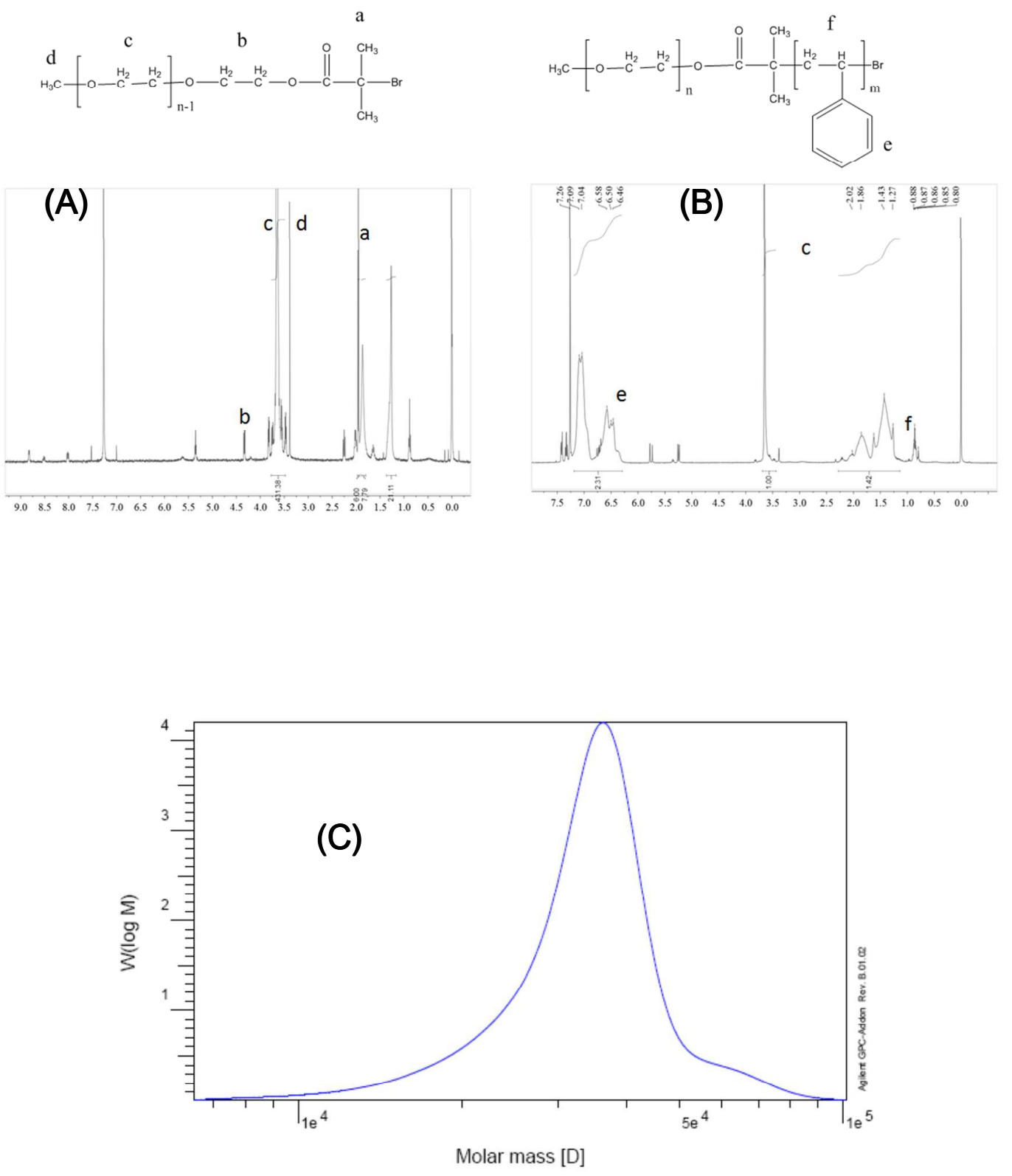
Figure S1. ${ }^{1} \mathrm{H}$ NMR spectra of (A) PEO-Br and (B) the synthesized diblock copolymer PEO-b-PS. (C) The gel permeation chromatograph (GPC) trace of diblock copolymer PEO- $b$-PS. 


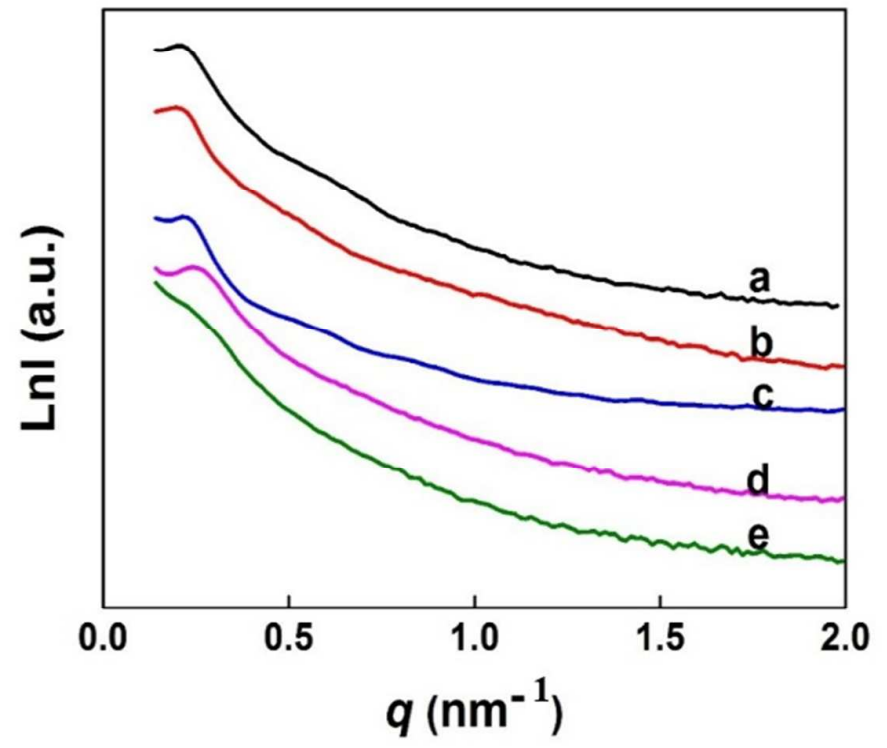

Figure S2. SAXS patterns of the $\mathrm{CoO}_{\mathrm{x}} / \mathrm{C}-n-600$ nanocomposites: (a) $\mathrm{CoO}_{\mathrm{x}} / \mathrm{C}-0.1-600$,

(b) $\quad \mathrm{CoO}_{\mathrm{x}} / \mathrm{C}-0.15-600$,

(c) $\mathrm{CoO}_{\mathrm{x}} / \mathrm{C}-0.2-600$,

(d) $\quad \mathrm{CoO}_{\mathrm{x}} / \mathrm{C}-0.25-600$,

$\mathrm{CoO}_{\mathrm{x}} / \mathrm{C}-0.5-600$. 


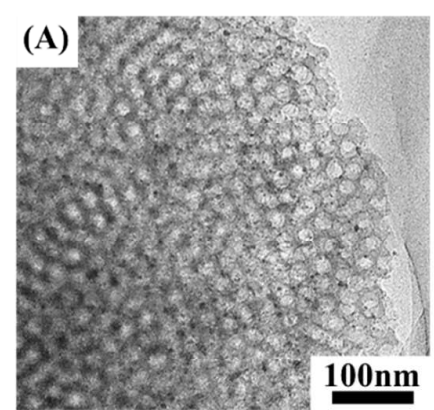

(D)

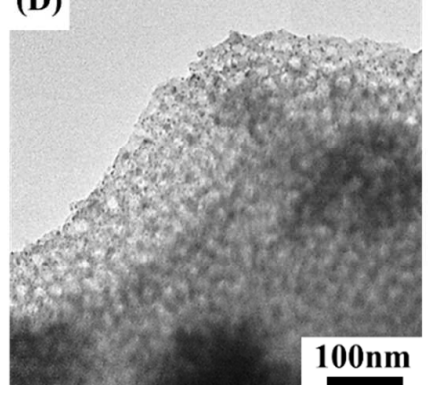

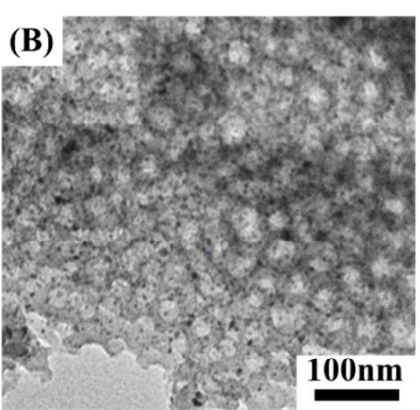

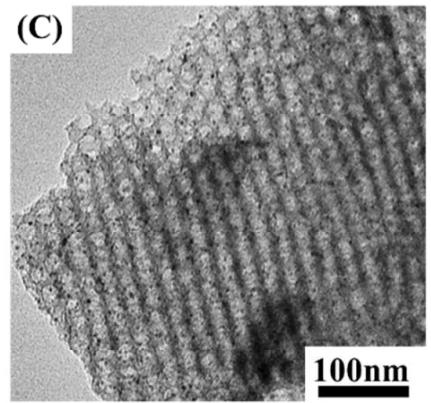

(E)

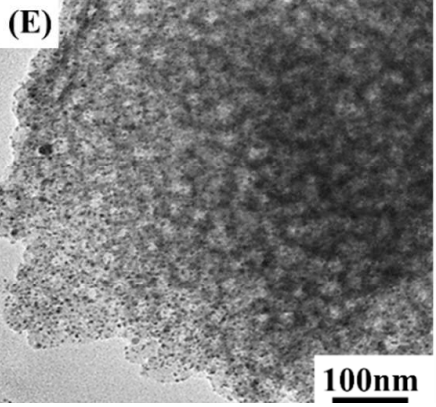

Figure S3. The TEM images of $\mathrm{CoO}_{\mathrm{x}} / \mathrm{C}-n-600$ nanocomposites: (A) $\mathrm{CoO}_{\mathrm{x}} / \mathrm{C}-0.1-600$,

(B) $\quad \mathrm{CoO}_{\mathrm{x}} / \mathrm{C}-0.15-600$,

(C) $\mathrm{CoO}_{\mathrm{x}} / \mathrm{C}-0.2-600$,

(D) $\quad \mathrm{CoO}_{\mathrm{x}} / \mathrm{C}-0.25-600$,

(E) $\mathrm{CoO}_{\mathrm{x}} / \mathrm{C}-0.5-600$. 


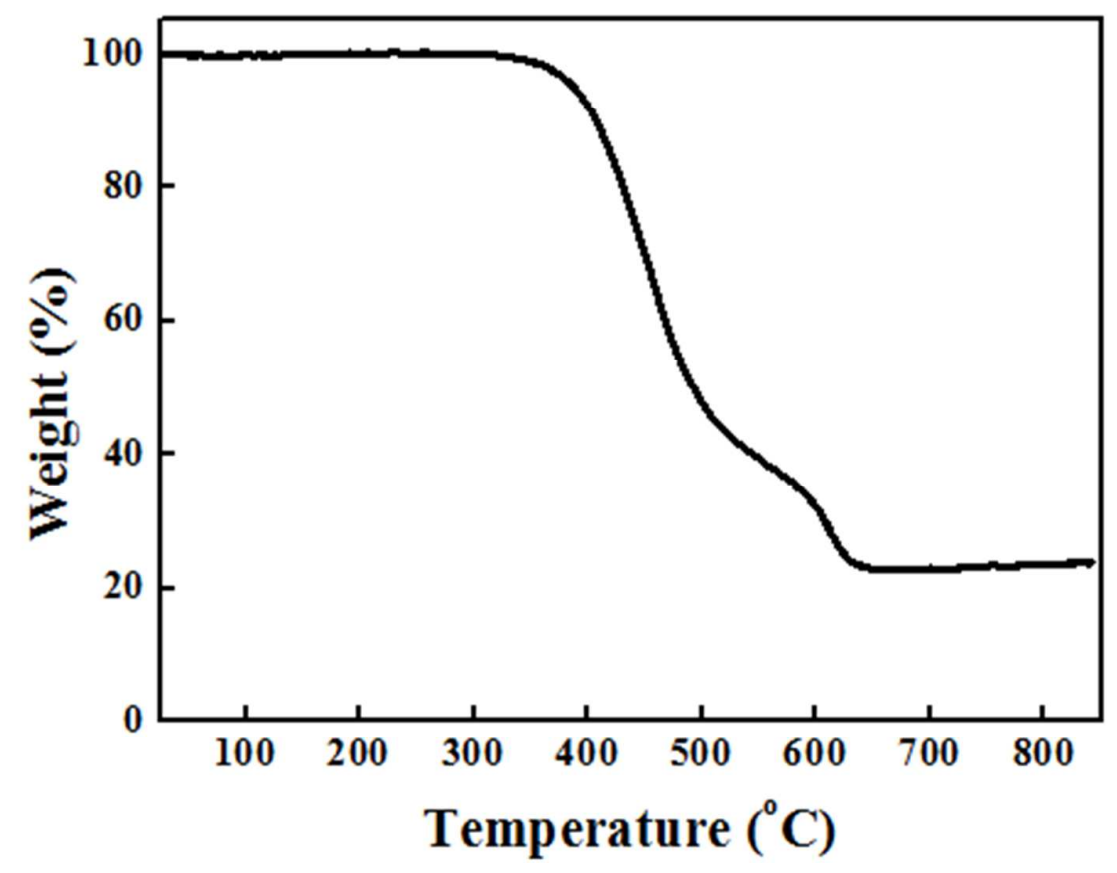

Figure S4. The TG curves of $\mathrm{CoO}_{\mathrm{x}} / \mathrm{C}-0.2-700$. The measurement was performed using a Mettler Toledo TGA/SDTA851 analyzer from 25 to $900{ }^{\circ} \mathrm{C}$ under air with a heating rate of $5^{\circ} \mathrm{C} / \mathrm{min}$. 
Table S1. Summary of graphitic degree of various representative carbon materials reported in literature.

\begin{tabular}{lccc}
\hline \multicolumn{1}{c}{ material } & $\begin{array}{c}\text { pyrolysis } \\
\text { temperature }\end{array}$ & $\mathbf{I}_{\mathbf{D}} / \mathbf{I}_{\mathbf{G}}$ & reference \\
\hline porous graphitic carbon & $900{ }^{\circ} \mathrm{C}$ & 1.2 & 1 \\
ordered mesoporous carbon & $900{ }^{\circ} \mathrm{C}$ & 1.1 & 2 \\
carbon nano-onions & $1650{ }^{\circ} \mathrm{C}$ & 1.71 & 3 \\
ordered mesoporous carbon & $900{ }^{\circ} \mathrm{C}$ & 1.0 & 4 \\
graphitic mesoporous carbon & $900{ }^{\circ} \mathrm{C}$ & 0.98 & 5 \\
Few layer graphene frameworks & $1000{ }^{\circ} \mathrm{C}$ & 0.65 & 6 \\
ordered mesoporous carbon & $800{ }^{\circ} \mathrm{C}$ & 1.12 & This work. \\
\hline
\end{tabular}

\section{References}

1. Wang, Z.L.; Zhang, X.B.; Liu, X.J.; Lv, M.F.; Yang, K.Y.; Meng, J. Co-gelation synthesis of porous graphitic carbons with high surface area and their applications. Carbon 2011, 49, 161-169.

2. Gokulakrishnan, N.; Kania, N.; Léger, B.; Lancelot, C.; Grosso, D.; Monflier, E.; Ponchel, A. An ordered hydrophobic P6mm mesoporous carbon with graphitic pore walls and its application in aqueous catalysis. Carbon 2011, 49, 1290-1298.

3. Plonska-Brzezinska, M.; Molina-Ontoria, A.; Echegoyen, E. Post-modification by low-temperature annealing of carbon nano-onions in the presence of carbohydrates. Carbon 2014, 67, 304-317.

4. Joglekar, M.; Pylypenko, S.; Otting, M.; Valenstein, J.; Trewyn, B. Universal and Versatile Route for Selective Covalent Tethering of Single-Site Catalysts and Functional Groups on the Surface of Ordered Mesoporous Carbons. Chem. Mater. 2014, 26, 2873-2882.

5. Liu, M.Z.; Wang, C.; Wang; X. Interface-facilitated hydrothermal synthesis of sub-micrometre graphitic carbon plates. J. Mater. Chem., 2012, 22, 17820-17826.

6. Jiao, Y.C.; Han, D.D.; Liu, L.M.; Ji, L.; Guo, G.N.; Hu, J.H.; Yang, D.; Dong, A.G. Highly Ordered Mesoporous Few-Layer Graphene Frameworks Enabled by $\mathrm{Fe}_{3} \mathrm{O}_{4}$ Nanocrystal Superlattices. Angew. Chem. Int. Ed. 2015, 14, 5727-5731. 